\title{
Load Balancing in Distributed Computing Over Wireless LAN: Effects of Network Delay
}

\author{
S. Dhakal, M. M. Hayat, M. Elyas, J. Ghanem, and C. T. Abdallah \\ Department of Electrical and Computer Engineering, University of New Mexico \\ Albuquerque, NM 87131-0001 USA; E-Mail: \{dhakal,hayat,elyas,jean,chaouki\}@ece.unm.edu
}

\begin{abstract}
A complete analytical solution to one-shot load balancing in a two-node distributed system is presented and verified experimentally. The model takes into account uncertainty in network delays and the variability in the processors' speeds. The system consisting of two Transmeta processors connected through a wireless LAN, utilizing an $802.11 b$ access point, is used as the testbed. The statistics of the network delays are estimated experimentally and used in the analytical model to show the effect of the balancing instant and gain on the overall completion times of various workloads. The analytical results are verified by comparing them to actual experiments and Monte-Carlo simulation. The effect of the communication delay on the choice of the gain and the balancing instant is clearly observed. Finally, the model is used to determine the optimal gain and balancing instant for different initial load distributions.
\end{abstract}

\section{INTRODUCTION}

Dynamic load balancing is the distribution of computational loads across all the available computational elements (CEs) during run-time. Any effective balancing policy requires some amount of communication and load migration between the participant CEs. Therefore, the policy depends on a number of factors like knowledge of the state of individual CEs, the network topology, processing capacity of each $\mathrm{CE}$, etc. Specifically, in an unpredictable shared environment such as the wireless LAN, the variation in the network behavior is significant. Firstly, such network typically exhibit significant latency in the exchange of information and the actual transfer of loads. Thus, the balancing policy has no choice but to rely on dated information about the system of nodes, which, in turn, may result in unnecessary transfer of loads while certain CEs remain idle as the loads are in transit. Most of the existing load-balancing policies take into account this time-delay factors of the physical medium [3], [4], [5]. Secondly and more importantly, the delay fluctuations in wireless networks are random in nature and are also sensitive to the size of the load to be transferred. This introduces uncertainty in the shared knowledge among the nodes, and hence, any load-balancing policy designed for a deterministic-delay environment may be subject to failures.

The limitations and the overheads in the implementation of the deterministic-delay load-balancing policy in a random delay environment has been discussed by the authors in [6]. Similarly, in [7], [8], Monte-Carlo studies and real-time experiments were conducted over the wireless LAN to study a one-shot dynamic load-balancing scheme for distributed systems. The results indicate that in such an unpredictable environment, the load-balancing problem has to be tackled in a statistical framework to maximize the resource utilization. It is observed that there is an interplay between the stochastic delay (e.g., its mean and variance, as well as its dependence on the random load to be transferred) and the strength and frequency of balancing. Indeed, our earlier work has motivated us to look into dynamic load balancing as an optimization problem. In particular, we have observed that in a delay-limited environment and for a given initial load distribution among the available CEs, there exist an optimal load-balancing instant and an optimal balancing gain associated with a one-shot load-balancing policy for which the overall completion time of a workload is minimized.

Previously, we presented a regeneration-theory-based mathematical model, represented by a set of coupled integrodifference equations, for one-shot load-balancing policy as applied to a two-nodes distributed system [8], [10]. The model characterizes the mean of the overall completion time as a function of the balancing instant. In this paper, we present a complete solution to the model and validate the predictive ability of it by comparing its results to that from experiments and Monte Carlo simulation. We first conduct experiments to empirically calculate the network parameters in the wireless LAN using an $802.11 \mathrm{~b}$ access point, and then utilize them in the mathematical model to design an optimal one-shot load-balancing policy. The model is then used to calculate the optimal balancing instant and the balancing gain for different initial workload distributions. In this paper, a task is the smallest (indivisible) unit of workload and load (or workload) is the collection of tasks.

\section{DESCRIPTION OF THE LOAD-BALANCING POLICY}

The queuing model that characterizes the stochastic dynamics of the load-balancing problem is detailed in [6]. We provide a brief review of it for completeness. If $Q_{i}(t)$ is the queue length of the $i$ th node at time $t$, then after time $\Delta t$, the queue length increases due to the arrival of external tasks $J_{i}(t, t+\Delta t)$ at node $i$ as well as due to the arrival of tasks that have been sent to node $i$ by other nodes in the system at their load-balancing instants. Also, its queue length decreases as $C_{i}(t, t+\Delta t)$ tasks get served at node $i$ during the same interval and if there is any load-balancing instant during $[t, t+\Delta t)$, node $i$ might send a number of tasks to the other nodes in the system. In differential form (in $\Delta t$ time 
increments), the queue length is given as:

$$
\begin{aligned}
Q_{i}(t+\Delta t) & =Q_{i}(t)-C_{i}(t, t+\Delta t) \\
& +J_{i}(t, t+\Delta t)-\sum_{j \neq i} \sum_{l} L_{j i}(t) I_{\left\{t_{l}^{i}=t\right\}} \\
& +\sum_{j \neq i} \sum_{k} L_{i j}\left(t-\tau_{i j, k}\right) I_{\left\{t_{k}^{j}=t-\tau_{i j, k}\right\}},
\end{aligned}
$$

where, $I_{A}$ is an indicator function for the event $A,\left\{t_{k}^{i}\right\}$ is a sequence of load-balancing instants for the $i$ th node, $C_{i}(t, t+\Delta t)$ is a Poisson process (with rate $\lambda_{i}$ ) describing the random number of tasks completed in the interval $[t, t+\Delta t)$, and $\tau_{i j, k}$ is the delay in transferring load $L_{i j}\left(t-\tau_{i j, k}\right)$ from node $j$ to node $i$ at the $k$ th load-balancing instant of node $j$. For the one-shot load-balancing policy, $t_{l}^{i}=\infty$ for all $l \geq 2$ and all $i \geq 1$. Further, we consider only the initial-value problem in this paper, and therefore, $\forall t>0, J_{i}(t, t+\Delta t)=$ 0 , i.e., there is no external tasks arrival in the distributed system of nodes. In an $n$-node system, for any $k \neq l$, the random load $L_{k l}(t)$ diverted from node $l$ to node $k$ is given by (2), where for any $j \neq k, \eta_{k j}=\eta_{j k}$ is the communication delay between the $k$ th and $j$ th nodes (with the convention $\left.\eta_{i i}=0\right)$. More precisely,

$$
L_{k l}(t)=\left\{\begin{array}{l}
K p_{k l}\left(Q_{l}(t)-n^{-1} \sum_{j=1}^{n} Q_{j}\left(t-\eta_{l j}\right) u\left(t-\eta_{l j}\right)\right) \\
u\left(Q_{l}(t)-n^{-1} \sum_{j=1}^{n} Q_{j}\left(t-\eta_{l j}\right) u\left(t-\eta_{l j}\right)\right) \\
k \neq l, \eta_{l k}(t) \leq t \\
K p_{k l} Q_{l}(t)\left(1-\frac{1}{n}\right), \text { otherwise }
\end{array}\right.
$$

where $u(\cdot)$ is the unit step function and $K \in[0,1]$ is the user-specified load-balancing gain (assumed to be the same for all nodes). The $l$ th node simply compares its load to the average over all load and sends out a fraction $p_{k l}$ of its excess load to the $k$ th node. (Of course, $\sum_{k \neq l} p_{k l}=1$ and $p_{l l}=0$ ). Finally, the fractions $p_{k l}$ are defined as:

$$
p_{k l}= \begin{cases}\frac{1}{n-2}\left(1-\frac{Q_{k}\left(t-\eta_{l k}\right)}{\sum_{i \neq l} Q_{i}\left(t-\eta_{l i}\right)}\right), & k \neq l, \eta_{l k}(t) \leq t \\ \frac{1}{n-1}, & \text { otherwise }\end{cases}
$$

where $n \geq 3$. A node sends a larger fraction of its excess load to a node with a small load relative to all other candidate recipient nodes. In this paper, we only consider the special case with $n=2$ in which case $p_{k l}=1$ whenever $k \neq l$.

\section{EXPERIMENTAL CHARACTERIZATION OF NETWORK DELAYS}

The delays in wireless LANs with infrastructure or wireless ad-hoc networks vary greatly and may severely affect any load-balancing outcome. To demonstrate and understand such variability, we will first present a delay-probing experiment conducted on the wireless networks. Our main focus will be to find the probability density function (pdf) of $\eta_{i j}$ (the communication delay from node $j$ to node $i$ ) and $\tau_{i j}$ (the load-transfer delay from node $j$ to node $i$ ). It has been observed in [8], [10] that $\tau_{i j}$ is dependent on the load size. Further, as will be discussed in detail in Sections V, $\tau_{i j}$ has an effect on the choice of optimal gain $K$ while $\eta_{i j}$ changes the optimal balancing instant $t_{b}$. In Section IV this delay distribution is integrated into the mathematical model as well as the simulator to validate the regenerationbased stochastic model of the one-shot load balancing. The wireless testbed consists of two $1 \mathrm{GHz}$ Transmeta Processor machines communicating with each other through an $802.11 \mathrm{~b}$ access point (AP). Both the nodes were connected to the same AP located on the same floor in the UNM EECE building, where other users and researchers were possibly using the network (wireless or wired). Each node sends to the other node a small time-stamped UDP packet carrying information about the number of tasks. The delay involved in this transaction is referred to as communication delay, which will later play a significant role in the performance of load-balancing policy. These experiments were run enough times and the histogram approximation of the pdf of the delays is plotted as shown in Fig. 1. The plot can reasonably be approximated by an exponential distribution with mean $0.0886 \mathrm{~s}$. To model the load-transfer delay, the nodes were made to transmit actual tasks between them, where each task (uniformly and independently chosen between $1 \mathrm{~KB}$ to $10 \mathrm{~KB}$ in size) comprised of a row of fixed number of elements, and total tasks transferred at a time is termed as load. When a node receives an entire batch of tasks it sends back a 3 Bytes (ACK) acknowledgement on the same established connection. The transfer delay is calculated from the application layer's perspective by taking the difference between the time the connection was established by a node for load transfer and the time it received the ACK packet. The actual load-transfer delay in transferring these tasks was normalized by the number of tasks being transferred to obtain the histogram approximation of the pdf of the transfer delay per task, which is shown in Fig. 2. This can also be well approximated by an exponential distribution with mean $0.3462 \mathrm{~s}$. The pdf represents the delay associated with the entire load rather than that of the TCP packets. The data for Figs. 1 to 3 were collected during the entirety of the load balancing experiments conducted for this paper.

In Fig. 3, the empirical mean of the transfer delay is plotted versus the actual number of tasks being transferred. Clearly, up to some threshold, the average delay is independent of the load size $L$ and thereafter increases monotonically with the size. We capture this behavior of the average transfer delay through the following parametric model [6]:

$$
\theta_{i j}=d_{\min }-\frac{1+e^{1 / L d \beta}}{1-e^{1 / L d \beta}}
$$

where $\theta_{i j}$ is the expected value of $\tau_{i j}$ (which follows exponential distribution), $d_{\min }$ is the minimum possible transfer 


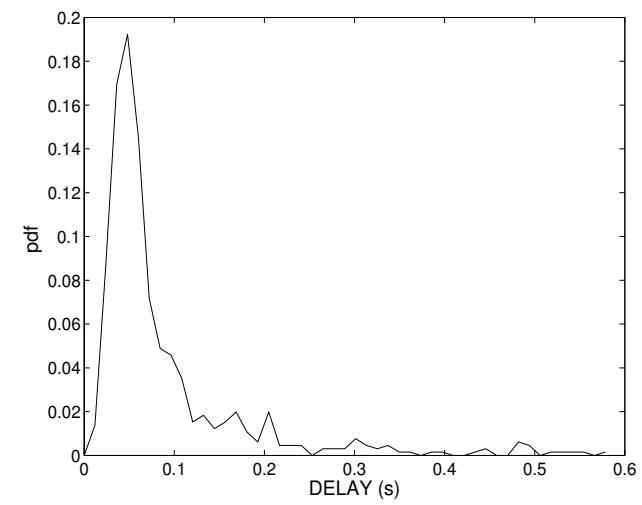

Fig. 1. Empirical pdf of the communication delay in an AP network.

delay, and $d$ and $\beta$ are constants. All of these parameters are estimated through data fitting and the solid-curve shown in Fig. 3 is generated according to the following parameters: $d_{\min }=0.3462 \mathrm{~s}, d=0.4$ and $\beta=0.1$.

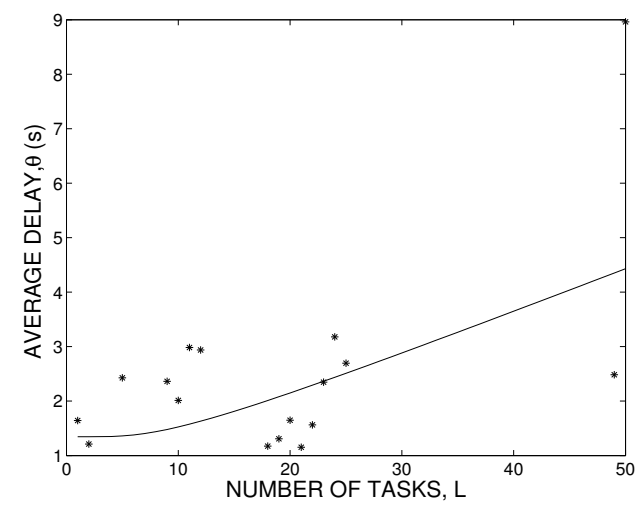

Fig. 3. Average transfer delay changes with the size of the load. The stars represent the empirical estimate of the delay while the solid line is its approximation using the delay model given by (4).

\section{A Regeneration-Principle-BASED Queuing MODEL FOR ONE-SHOT LOAD BALANCING}

The concept of regeneration has proven to be a powerful tool in the analysis of complex stochastic systems [1], [2], [9]. The idea of our approach is to define certain initial events, called regeneration events, defined as the completion of a task by any node or the arrival of a communication by any node. The key feature of these events is that their occurrences will regenerate queues that have similar statistical properties and dynamics as their predecessors. We assume that initially all queues have zero knowledge about the state of the other queues. The point here is that immediately after the occurrence of the initial event, we will have a set of new queues whose stochastic dynamics are identical to the original queues. However, they will have a different set of initial conditions. Namely, we will have a different initial load distribution if the initial event is a task completion, or

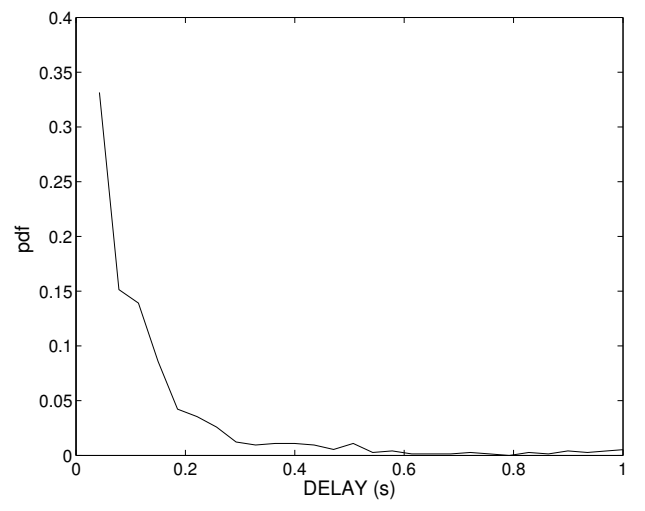

Fig. 2. Empirical pdf of the load-transfer delay in an AP network.

a different knowledge state if the initial event happens to be a communication arrival rather than a task completion. The knowledge state is defined formally for the two-node setup as follows (a more general definition is given in [10]): Depending upon the choice of the balancing instant, any node may or may not receive a communication from the other node. We assign a scalar variable to each of the two nodes and initially set the values to 0 (corresponding to the null knowledge state). If a communication arrives from any of the nodes, the variable corresponding to that particular node is set to 1 . For example, state $(1,0)$ corresponds to the case when only node 1 knows about node 2 .

To see how the idea of regeneration works, consider the example for which the initial event happens to be the departure of a task at node 1 . In this case, the system (of queue) dynamics remains unchanged except that node 1 will now have 1 task less from its initial workload. Thus, upon the occurrence of this particular initial event, the queues have re-emerged with a different initial load. A similar behavior is observed if the initial event is the arrival of a communication to one of the nodes in which case the newly emerged queues will have a new knowledge state.

We now present the novel analytical model for oneshot load balancing. It is assumed that each node has an exponential service time with parameters $\lambda_{D_{1}}$ and $\lambda_{D_{2}}$, respectively. As discussed in Section III, the communication delays from node 1 to node 2 and from node 2 to node 1 are also assumed to follow an exponential distribution with rates $\lambda_{21}$ and $\lambda_{12}$, respectively. Let $W, X, Y$ and $Z$ be the events representing task completion at node 1 , task completion at node 2 , communication arrival at node 2 and communication arrival at node 1 , respectively. Let $T$ be the regeneration event defined by $T=\min (W, X, Y, Z)$. Let $\mu_{m, n}^{k_{1}, k_{2}}\left(t_{b}\right)$ be the expected value of the overall completion time given that the balancing is executed at time $t_{b}$, where nodes 1 and 2 are assumed to have $m$ and $n$ tasks at time $t=0$, and the system knowledge state is $\left(k_{1}, k_{2}\right)$ at time $t=0$. Suppose that the initial event happens to be the departure of a task at node 1 at 
time $t=s, 0 \leq s \leq t_{b}$. At this instant, the system dynamics remains the same except that node 1 will now have $m-1$ tasks. Thus, the queue has re-emerged (with a different initial load, nonetheless) and the average of the overall completion time is now $s+\mu_{m-1, n}^{k_{1}, k_{2}}\left(t_{b}-s\right)$. The other possibilities of the initial event are taken into account similarly. Now, the dynamics of $\mu_{m, n}^{k_{1}, k_{2}}\left(t_{b}\right)$ can be characterized by the following (5) set of four (one for each initial information state) coupled integro-difference equations [8], [10]:

$$
\begin{array}{cc} 
& \mu_{m, n}^{k_{1}, k_{2}}\left(t_{b}\right)=\int_{t_{b}}^{\infty} f_{T}(s)\left[\mu_{m, n}^{k_{1}, k_{2}}(0)+t_{b}\right] d s \\
+ & \int_{0}^{t_{b}} f_{T}(s)\left[\mu_{m-1, n}^{k_{1}, k_{2}}\left(t_{b}-s\right)+s\right] \mathrm{P}\{T=W\} d s \\
+ & \int_{0}^{t_{b}} f_{T}(s)\left[\mu_{m, n-1}^{k_{1}, k_{2}}\left(t_{b}-s\right)+s\right] \mathrm{P}\{T=X\} d s \\
+\quad & \int_{0}^{t_{b}} f_{T}(s)\left[\mu_{m, n}^{k_{1}, 1}\left(t_{b}-s\right)+s\right] \mathrm{P}\{T=Y\} d s \\
+ & \int_{0}^{t_{b}} f_{T}(s)\left[\mu_{m, n}^{1, k_{2}}\left(t_{b}-s\right)+s\right] \mathrm{P}\{T=Z\} d s,
\end{array}
$$

where $\left(k_{1}, k_{2}\right) \in\{0,1\}^{2}$. This can further be simplified into a set of difference-differential equations (6):

$$
\begin{gathered}
\frac{d \mu_{m, n}^{k_{1}, k_{2}}\left(t_{b}\right)}{d t_{b}}=\lambda_{D_{1}} \mu_{m-1, n}^{k_{1}, k_{2}}\left(t_{b}\right)+\lambda_{D_{2}} \mu_{m, n-1}^{k_{1}, k_{2}}\left(t_{b}\right) \\
+\lambda_{21} \mu_{m, n}^{k_{1}, 1}\left(t_{b}\right)+\lambda_{12} \mu_{m, n}^{1, k_{2}}\left(t_{b}\right)-\lambda \mu_{m, n}^{k_{1}, k_{2}}\left(t_{b}\right)+1
\end{gathered}
$$

Each of these equations involves a recursion, which have been exploited to solve them by writing an efficient MATLAB program. We also point out that while solving each of these equations, we need to solve for their corresponding initial conditions, namely, $\mu_{m, n}^{k_{1}, k_{2}}(0)$. We next turn to calculating these initial conditions.

We first present a methodology to calculate $\mu_{m, n}^{1,1}(0)$, where $m \geq n$. According to (2) with $p_{21}=1, L_{21}(0)$ is

$$
L_{21}(0)=\left\lfloor\frac{K(m-n)}{2}\right\rfloor=L .
$$

If $L$ tasks are transferred from node 1 to node 2 then node 1 has $m-L$ tasks at time $t=0$. If $T_{1}$ is the waiting time before all the tasks are served, then the cumulative distribution function (cdf) of $T_{1}$ is that of an Erlang random variable since the inter-departure times are assumed to be independent. More precisely,

$$
F_{T_{1}}\left(t_{1}\right)=\left(1-\sum_{x=0}^{m-L-1} e^{-\lambda_{D_{1}} t_{1}} \frac{\left(\lambda_{D_{1}} t_{1}\right)^{x}}{x !}\right) u\left(t_{1}\right) .
$$

As discussed in Section III the load-dependent random transfer delay $\tau_{21}$ is assumed to follow an exponential pdf with rate $\lambda_{t}$, which is a function of $L$ as given by (4), where $\theta_{21}=\frac{1}{\lambda_{t}}$. Therefore, the cdf of the total completion time of node 2 , denoted as $T_{2}$, is calculated from (7) as [10]:

$$
\begin{gathered}
F_{T_{2}}\left(t_{2}\right)=1-e^{-\lambda_{t} t_{2}} \\
-\lambda_{t} e^{-\lambda_{D_{2}} t_{2}}\left[\sum_{r=0}^{n-1} \sum_{x=L}^{L+n-r-1} \frac{\left(\lambda_{D_{2}}\right)^{r}}{r !} \frac{\left(\lambda_{D_{2}}\right)^{x}}{x !} g\left(t_{2} ; r ; x\right)\right] \\
-\lambda_{t} e^{-\lambda_{D_{2}} t_{2}}\left[\sum_{x=0}^{L-1} \frac{\left(\lambda_{D_{2}}\right)^{x}}{x !} g_{1}\left(t_{2} ; 0 ; x\right)\right] \\
\text { where } g\left(t_{2} ; r ; x\right)=\int_{0}^{t_{2}} t^{r}\left(t_{2}-t\right)^{x} e^{-\lambda_{t} t} d t \\
=\sum_{k=0}^{x}(-1)^{k} \frac{t_{2}{ }^{x-k} x !}{(x-k) ! k !} \frac{(r+k) !}{\lambda_{t}^{r+k+1}}\left[1-e^{\lambda_{t} t_{2}} \sum_{j=0}^{r+k} \frac{\left(\lambda_{t} t_{2}\right)^{j}}{j !}\right], \\
g_{1}\left(t_{2} ; 0 ; x\right)=\int_{0}^{t_{2}}\left(t_{2}-t\right)^{x} e^{-\left(\lambda_{t}-\lambda_{D_{2}}\right) t} d t
\end{gathered}
$$

Now, the overall completion time is $T_{C}=\max \left(T_{1}, T_{2}\right)$, and recall that its average $\mathrm{E}\left[T_{C}\right]$ is $\mu_{m, n}^{1,1}(0)$. By exploiting the independence of $T_{1}$ and $T_{2}$ we obtain

$$
\mu_{m, n}^{1,1}(0)=\int_{0}^{\infty} t\left[f_{T_{1}}(t) F_{T_{2}}(t)+F_{T_{1}}(t) f_{T_{2}}(t)\right] d t .
$$

In the case when $L=0$, no load transfer occurs and the calculation of $\mu_{m, n}^{1,1}(0)$ becomes much simpler. In particular, it can be shown that

$$
\mu_{m, n}^{1,1}(0)= \begin{cases}\frac{m}{\lambda_{D_{1}}}+\frac{n}{\lambda_{D_{2}}} & \\ -\frac{\left(\lambda_{D_{1}}\right)^{m}}{(m-1) !} \sum_{x=0}^{n-1} \frac{(m+x) !}{\left(\lambda_{D_{1}}+\lambda_{D_{2}}\right)^{m+x+1}} \frac{\left(\lambda_{D_{2}}\right)^{x}}{x !} & \\ -\frac{\left(\lambda_{D_{2}}\right)^{n}}{(n-1) !} \sum_{x=0}^{m-1} \frac{(n+x) !}{\left(\lambda_{D_{1}}+\lambda_{D_{2}}\right)^{n+x+1}} \frac{\left(\lambda_{D_{1}}\right)^{x}}{x !}, & n>0 \\ \frac{m}{\lambda_{D_{1}}}, & n=0\end{cases}
$$

The calculations of $\mu_{m, n}^{0,0}(0), \mu_{m, n}^{0,1}(0)$ and $\mu_{m, n}^{1,0}(0)$ can be performed in a similar fashion. In this case, $L_{i j}(0)$ is first computed according to (2) corresponding to each knowledge state. For example, while calculating $\mu_{m, n}^{1,0}(0)$, we need to find $L_{21}(0)=\left\lfloor\frac{K(m-n)}{2}\right\rfloor$ as well as $L_{12}(0)=\left\lfloor\frac{K n}{2}\right\rfloor$.

\section{RESUlts}

The load-balancing experiments were conducted over a wireless network using an $802.11 \mathrm{~b}$ AP. The testing was completed on two $1 \mathrm{GHz}$ Transmeta Processor machines. We consider the case where both nodes execute the load balancing at a common balancing time $t_{b}$. The application used to illustrate the load-balancing process was matrix multiplication, where one task has been defined as the multiplication of one row by a static matrix duplicated on both the nodes. The arithmetic precision of each element in a row was picked randomly from a specified range which made the execution time of a task variable. As for the communication part of the program, UDP was used to exchange queue size information among the nodes and TCP was used to transfer the data or tasks from one machine to another. The loadbalancing policy used in the experiments is governed by the 
two policies given in (2), where the node decides to use either one according to its knowledge states. Node 1 was assigned 100 tasks and node 2 was assigned 50 tasks.

Our first objective was to perform optimization over the balancing instant $t_{b}$ by fixing the balancing gain at $K=$ 1. The result is as shown in Fig. 4 where each point is averaged over 20 experiments for a given balancing instant. The average communication delay was found to be $0.1012 \mathrm{~s}$ and the average service time per task for node 1 was $0.7781 \mathrm{~s}$ while that for node 2 was $0.8315 \mathrm{~s}$. Using these mean values in conjunction with the exponential-pdf approximation undertaken in our analytical model, we generated graphs of the average completion time as a function of the balancing instant, as shown in Fig. 5. Both experimental and theoretical results show that if the load balancing is performed blindly, i.e., at $t_{b}=0$ (as in the onset of receiving the initial load), the performance is very sensitive to the balancing instant. Even a small amount of delay in the execution of load balancing will increase the completion time substantially. This sudden rise in the completion time is due to the hybrid knowledge states in the system. That is, a node may know the queue size of the other node while the other node may not know its queue size due to random communication delay. This results in the situation where one node uses blind load-balancing action while the other node takes an informed balancing action, in accordance with the policy given by (2). Consequently, this may result in a yet more severe uneven distribution of the load than the initial distribution. On the other hand, the completion time drops significantly to approximately $64 \mathrm{~s}$ if the load balancing is executed after the average arrival time of the communications so that both nodes undertake informed load balancing. These characteristics, which are now predicted by our analytical model for the first time, have been previously reported using the simulation and the experiments [7], [8], [10].

Next, we conducted the experiments by fixing $t_{b}$ at $100 \mathrm{~ms}$ and performed the optimization over the balancing gain $K$. The plot generated from experimental data is shown in Fig. 6, where 20 runs were made for each gain value. The empirically estimated average communication delay was $0.1190 \mathrm{~s}$ and the average service time per task for node 1 and node 2 were $0.7540 \mathrm{~s}$ and $0.7847 \mathrm{~s}$, respectively. The average transfer delay per task was estimated as $0.2201 \mathrm{~s}$. Using these empirical estimates of the delay parameters in the analytical model and the Monte-Carlo simulator, we generated the plot shown in Fig. 7. The optimal performance occurs at $K=0.9$ according to the experiments while both the simulator and analytical model yield an optimal gain of $K=0.6$. This discrepancy is due to two factors: First, due to time constraints, the experiments were run only 20 times per gain value. Second, the approximation in the loaddependent delay model [as described by (4)] may have not accurately represented the true variability, as a function of the actual load size, in the load-transfer delay. Nonetheless, it is
TABLE I

EXPERIMENTAL RESULTS FOR THE THEORETICALLY DETERMINED OPTIMAL LOAD BALANCING

\begin{tabular}{|c|c|c|c|c|}
\hline $\begin{array}{c}\text { Initial } \\
\text { workload } \\
(\mathrm{m}, \mathrm{n})\end{array}$ & $\begin{array}{c}\text { optimal } \\
K\end{array}$ & $\begin{array}{c}\text { optimal } \\
t_{b} \\
(\mathrm{~s})\end{array}$ & $\begin{array}{c}\mu_{m, n}^{0,0} \\
\text { (s) }\end{array}$ & $\begin{array}{l}\text { Average Completion } \\
\text { Time (Experiment) } \\
\text { (s) }\end{array}$ \\
\hline$(100,50)$ & 0.9 & 5 & 61.7 & 62.53 \\
\hline$(100,0)$ & $\overline{0.8}$ & 0 & 49.7 & 46.59 \\
\hline$(20,40)$ & 1 & 5 & 25.3 & 25.78 \\
\hline$(70,50)$ & 0.9 & 4 & 49.58 & 48.53 \\
\hline$(50,50)$ & 1 & 7 & 41.78 & 40.08 \\
\hline
\end{tabular}

important to note that the optimal gain is less than 1 in all the cases, which implies that an appropriate conservative loadbalancing action outperforms a full-extent load balancing (corresponding to $K=1$ ). To have further insight into this behavior, we observe that since $t_{b}=100 \mathrm{~ms}$, the knowledge state of the nodes is most probably in a hybrid state [i.e., either $(1,0)$ or $(0,1)]$. Therefore, load balancing has to be done conservatively (i.e., $K<1$ ) to reduce the extent of aggravating the load imbalance due to the blind loadbalancing action of one of the two nodes.

We also repeated the load-balancing experiments to investigate the optimization over the balancing gain $K$ using $t_{b}=$ $2 \mathrm{~s}$. Here, the load balancing is executed most probably after the arrival of communication (since average communication delay was found to be 0.0675 s during the hours when these experiments were conducted). According to the experiment as well as the analytical and Monte-Carlo-based results, the optimal gain is found to be $K=1$. The intuitive explanation for this behavior is that the load balancing is done in an informed way. Therefore, node 1 should be allowed to migrate all its excess load to node 2 as decided by the policy.

Finally, we used the model to calculate optimal $K$ and the optimal $t_{b}$ for different initial load distribution that minimizes the expected value of the overall completion time. The model used empirical estimates of average communication delay, which was $0.0886 \mathrm{~s}$, and the average service time per task for node 1 and node 2 , which were $0.7597 \mathrm{~s}$ and $0.8036 \mathrm{~s}$, respectively. Then, the optimal $K$ and the optimal $t_{b}$ were incorporated in the actual experimental set-up to find the average overall completion time for each initial workload. The results are tabulated in Table I. It is interesting to note that even for a balanced initial load distribution given by $(m, n)=(50,50)$, there is an optimal gain and the optimal balancing instant, which is attributable to the difference in average speeds of the two processors.

\section{CONCLUSION}

The regeneration-theory based analytical model for oneshot load balancing has been verified by comparing the results to real-time load-balancing experiments performed over a two-node wireless-LAN network as well as MonteCarlo simulation. Our results confirm that for each initial distribution of workload and processing speed, there exist 


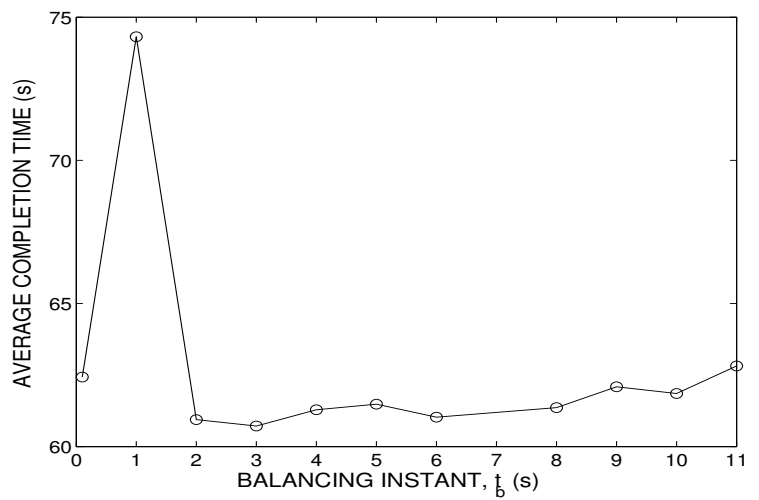

Fig. 4. Experimental average total task-completion time as a function of the balancing instant. The load-balancing gain parameter is set at $K=1$.

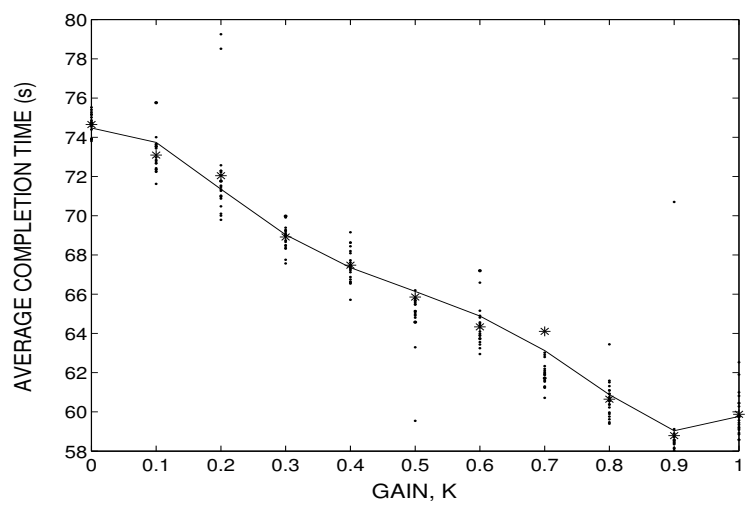

Fig. 6. Experimental average of the total task-completion time as a function of the balancing gain. The load-balancing instant is set at $t_{b}=100 \mathrm{~ms}$. Dots represent the actual completion time while stars represent their empirical mean.

an optimal load-balancing gain and an optimal balancing instant that minimize the average overall completion time of the initial workload. The model also confirms that in a delay-limited environment, the optimal performance of a load-balancing policy is attained when every node has knowledge of the workload of other nodes. Further, in such environments a conservative choice of the balancing gain may better suit uncertainties in processor speed and delay. This work is supported by the National Science Foundation under Information Technology Research (ITR) grants No. ANI-0312611 and ANI-0312182.

\section{REFERENCES}

[1] C. Knessly and C. Tiery,“Two tandem queues with general renewal input I: Diffusion approximation and integral representation," SIAM J. Appl. Math.,vol. 59, pp. 1917-1959, 1999.

[2] F. Bacelli and P. Bremaud, Elements of Queuing Theory: Palm-Martingale Calculus and Stochastic Recurrence. New York: Springer-Verlag, 1994.

[3] Z. Lan, V. E. Taylor, and G. Bryan, "Dynamic load balancing for adaptive mesh refinement application," Proc. ICPP'2001, Valencia, Spain, 2001.

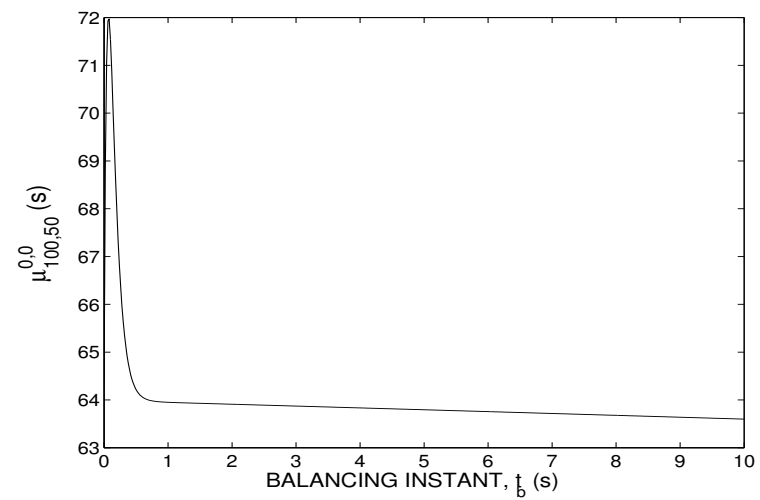

Fig. 5. The theoretical prediction of the graph in Fig. 4 using the regeneration model.

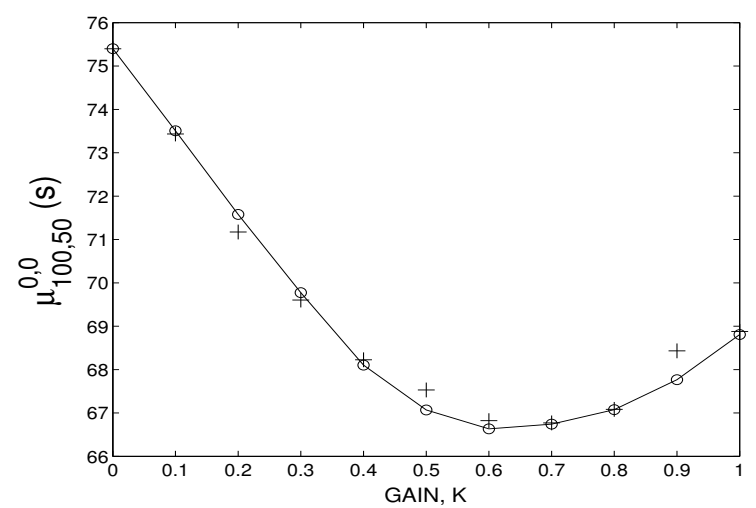

Fig. 7. Prediction of the results shown in Fig. 6. Circles represent the analytical results while crosses are evaluated according to Monte-Carlo simulation.

[4] T. L. Casavant and J. G. Kuhl, "A taxonomy of scheduling in general-purpose distributed computing systems," IEEE Trans. Software Eng., vol. 14, pp. 141-154, Feb. 1988.

[5] G. Cybenko, "Dynamic load balancing for distributed memory multiprocessors," IEEE Trans. Parallel and Distributed Computing, vol. 7, pp. 279-301, Oct. 1989

[6] M. M. Hayat et al., "Dynamic time delay models for load balancing. Part II: Stochastic analysis of the effect of delay uncertainty, Advances in Time Delay Systems," LNCSE vol. 38, pp. 355-368, Springer-Verlag, 2004.

[7] S. Dhakal et al., "Dynamical discrete-time load balancing in distributed systems in the presence of time delays," Proceedings of the IEEE Conference on Decision and Controls (CDC 2003), Maui, Hawaii, pp. 5128-5134, Dec 2003.

[8] J. Ghanem et al., "Load balancing in distributed systems with large time delays: Theory and experiment,"Proceedings of the IEEE/CSS 12th Mediterranean Conference on Control and Automation (MED '04), Aydin, Turkey, June 2004.

[9] D. J. Daley and D. Vere-Jones, An introduction to the theory of point processes. Springer-Verlag, 1988.

[10] S. Dhakal et al., "On the optimization of load balancing in distributed networks in the presence of delay, Advances in Communication Control Networks," LNCSE vol. 308, pp. 223-244, Springer-Verlag, 2004. 